\title{
Análisis del nivel de competitividad empresarial en el panorama publicitario colombiano fundamentada en el éxito creativo y la responsabilidad ética y jurídica ${ }^{1}$
}

\section{Analysis of the Level of Competitiveness Business in the Colombian Advertising Scene Based on Creative Success}

DOI: http://dx.doi.org/10.17981/juridcuc.13.1.2017.1

Artículo de investigación.

Fecha de recepción: 28/06/2017 Fecha de aceptación: 23/08/2017

\author{
Estela Núñez Barriopedro ${ }^{2}$ \\ Universidad de Alcalá (España) \\ estela.nunez@uah.es
}

\author{
Rafael Ravina Ripoll ${ }^{3}$ \\ Universidad de Cádiz (España) \\ rafael.ravina@uca.es
}

Para citar este artículo:

Núñez, E. y Ravina, R. (2017). Análisis del nivel de competitividad empresarial en el panorama publicitario colombiano fundamentada en el éxito creativo y la responsabilidad ética y jurídica. JURÍDICAS CUC, vol. 13, no. 1, pp. 9-28. DOI: http://dx.doi.org/10.17981/juridcuc.13.1.2017.1

\footnotetext{
${ }^{1}$ Este trabajo se encuentra dentro de los resultados de la investigación enmarcados en el proyecto I+D: Estudio multidisciplinar de la influencia de la creatividad y la felicidad corporativa en el desarrollo sostenible-económica, social y de medioambiente de los territorios. Bajo la dirección principal de: Luis Bayardo Tobar Pesantez (Universidad Politécnica Salesiana), Rafael Ravina Ripoll (Universidad de Cádiz), Ana Fondón Ludeña (Universidad de Extremadura), Antonio Rafael Fernández Paradas (Universidad de Granada) y Francisco Villena Manzanares (Universidad de Sevilla).

${ }^{2}$ Doctora en Ciencias económicas y empresariales por la Universidad de Alcalá y Licenciatura en Administración y dirección de empresas en dicha universidad. Ha recibido varios premios en docencia y en investigación. Es profesora acreditada en UAH, UC3M y UNIR, entre otras. Tiene numerosas publicaciones y es directora académica del MBA en la Universidad de Alcalá.

3 Doctor en Historia por la Universidad de Cádiz y Licenciatura en Ciencias económicas y empresariales en dicha universidad; Profesor de la Organización de Empresas de la Universidad de Cádiz. Es autor y coautor de artículos, trabajos en congresos internacionales y libros vinculados con la economía de la felicidad y la creatividad. Ha sido profesor invitado en la Universidad Autónoma de Baja California, la Business and Law Frankfurt University of Applied Sciences y la Universidad de Verona, donde ha impartido conferencias sobre happiness management.
}

- The author; licensee Universidad de la Costa - CUC. 


\section{Resumen}

Las agencias que participan en sistemas de evaluación competitiva acuden a los festivales de publicidad donde la creatividad es especialmente valorada. El objetivo de este trabajo es medir y analizar la posición actual de las agencias de publicidad colombianas en el Ranking Iberoamericano de Publicidad. Para ello, se evalúa la competitividad de las agencias a través de su nivel de creatividad y según una ponderación rigurosa de los premios que obtienen y los festivales en los que son galardonados. Asimismo, se estudia la tendencia y se realiza una clasificación de agencias según su posición y puntuación en el ranking de publicidad. El cálculo del valor de las agencias para una edición se hace sobre los premios recibidos en los dos años anteriores. Para el caso de Colombia, se analiza el ranking de agencias en 2014, 2015 y 2016 para una muestra total de 69, 98 y 73 agencias respectivamente en Colombia. Se observa que las mejor situadas son las agencias internacionales.

\section{Palabras claves}

Creatividad, Competitividad, Agencias publicitarias, Ranking, Tendencia.

\section{Abstract}

Advertising Agencies that participate in competitive evaluation systems go to the advertising festivals where creativity is especially valued. The aim of this work is to measure and analyze the current position of Colombian advertising agencies in the Iberoamerican Advertising Ranking. In order to this, the competitiveness of the agencies is evaluated through their level of creativity and according to a rigorous weighting of the prizes they get and the festivals in which they are awarded. Likewise, the trend is studied and a classification of agencies according to their position and score in the ranking of Advertising is made. The calculation of the value of the agencies for an edition is made on the prizes got at the two previous years. For the case of Colombia, we analyze the ranking of agencies 2014, 2015 and 2016 for a total sample of 69, 98 and 73 agencies respectively in Colombia.

\section{Keywords}

Creativity, Competitiveness, Advertising agencies, Ranking, Trend. 


\section{INTRODUCCIÓN}

Los rankings de publicidad nacen en los años 70 en Estados Unidos y Reino Unido (Kübler y Proppe, 2012). Actualmente continúan siendo un fenómeno anglosajón para listar y ordenar según su nivel de competitividad creativa las agencias de publicidad.

Encontrarse entre las primeras 10 agencias de publicidad de los principales listados de clasificación es uno de los objetivos principales de la mayor parte de las agencias (Repiso, Llorente-Barroso y García-García, 2013). Este objetivo es explicado fundamentalmente porque las principales marcas buscan promocionarse con el servicio de las agencias de publicidad mejor posicionadas en los rankings publicitarios (Butkys y Herpel, 1992; Helgesen, 1994). Este tipo de práctica se ha convertido en una tradición en la industria publicitaria (Ogilvy, 1963).

Tradicionalmente, el objeto de evaluación de estos listados han sido los ingresos de las agencias, aunque también se ha recurrido al uso de encuestas a expertos y, en menor medida, al uso de premios en festivales.

Debido a la ausencia de cifras fiables de ingresos, fiables de autónomos y agencias nacionales, la industria publicitaria prefiere cambiar su enfoque hacia la creatividad (Myers, 2004). Desde el punto de vista de la psicología, el término de la creatividad se fundamenta en la originalidad, la capacidad de adaptación a la realidad y el grado de elaboración. Desde el punto de vista del mercadeo, se basa en la novedad (Amabile 1988; Amabile 1994; Amabile, Conti, Coon, Lazenby y Herron, 1996), la significatividad (Goldenberg, Mazursky y Solomon 1999) y la originalidad (Staernberg y Todt, 1999). Una vez visto ese trinomio del concepto de creatividad, conviene advertir que en la actualidad existe a nivel global una tendencia normativa a regular las actividades de las agencias publicitarias con la finalidad de evitar prácticas delictivas que nazcan bajo el paraguas de potenciar holísticamente el recurso intangible citado anteriormente, es decir, la creatividad. 


\section{MARCO TEÓRICO}

La investigación en creatividad ha tenido una larga tradición en la psicología y el mercadeo. Mientras que la mayoría de los estudios desde el punto de vista del marketing se orientan hacia la efectividad, los estudios psicológicos se enfocan más en medir la creatividad (Haberland y Dacin, 1992).

En el campo de la psicología se pueden dividir los estudios en tres líneas de investigación: la primera es la identificación de las características de las personas creativas (Barron, 1955; Barron y Harrington, 1981; MacKinnon, 1987). La creatividad en estos estudios está basada fundamentalmente en tres características fundamentales: originalidad, capacidad de adaptación a la realidad y grado de elaboración. La segunda línea se enfoca principalmente en los factores que pueden influir o mejorar el proceso creativo (Bruner, 1962; Newell, Shaw y Simon, 1959). En estos estudios se introduce el concepto de "novedad" como característica fundamental de la creatividad. Finalmente, la tercera identifica las consecuencias o resultados de la creatividad (Besemer y O’Quin, 1986; Besemer y Treffinger, 1981). En esta línea, se señala como dimensiones de la creatividad, la utilidad, la oportunidad, la transformación y la condensación (Jackson y Messick, 1965), incluso exponen que la creatividad mejora el ambiente de trabajo (Amabile, Conti, Coon, Lazenby y Herron, 1996) o el desarrollo profesional (Amabile, Tighe, Hill y Hennessey, 1994; Andrews y Smith 1996; Mumford y Simonton, 1997); también se establece que incrementa las ventas (Moorman y Miner, 1997; Song y Montoya-Weiss, 2001) y mejora los productos y los procesos (Deshpande, Farley y Webster, 1993).

En cuanto a la investigación en el área del márquetin, se encuentran dos enfoques: el primer enfoque es el impacto de la creatividad en el desarrollo de los productos, es decir, los factores que pueden influir en la percepción de la creatividad de un anuncio (Sethi, Smith y Park, 2001; Pieters, Warlop y Wedel, 2002; Ang, Lee y Leong 2007; Kilgour y Koslowm 2009) y en cómo los consumidores perciben esa creatividad (Haberland y Dacin, 1992; Michell, 1984), mientras que el segundo enfoque 
se basa en el rendimiento de la creatividad (Im y Workman, 2004; Moorman y Miner, 1997). Este último se orienta a la eficiencia y explica las diferencias entre evaluar la creatividad de los anuncios y de las agencias y sus clientes (Nyilasy y Reid, 2009; Devinney, Dowling y Collins 2005; Koslow, Sasser y Riordan, 2003).

Otra área de investigación relativa a la creatividad publicitaria se enfoca en el impacto positivo de la creatividad de los anuncios en la efectividad de los mismos (Rossiter, 2008), en general, y en las ganancias de ventas, en particular (Bell, 1992; Bogart, Tolley y Orenstein, 1970; El- Murad y West 2003; Smith, MacKenzie, Yang, Buchholz y Darley, 2007). Asimismo, estos estudios analizan otras implicaciones de la creatividad en términos de ganancia en la cuota de mercado (Buzzell, 1964); el impacto de la creatividad publicitaria en el corto plazo y en el largo plazo (Jones, 1995); el incremento de la fidelidad de marca (Stone, Besser y Lewis, 2000), la mejora en la atención a la marca (Pieters, Warlop y Wedel, 2002), una mayor notoriedad de marca (Gibson, 1996), la relación entre afinidad hacia la marca y rendimiento de las ventas (Bergkvist y Rossiter, 2008), mejores índices de persuasión (Till y Baack, 2005), mejora en la percepción de marca (Dahlen, Rosengren y Torn, 2008); y finalmente, exploran los efectos positivos de la creatividad publicitaria en la imagen de marca y sus verdaderas mejoras (Li, Dou, Wang y Zhou, 2008).

Otros estudios sobre la eficiencia de la creatividad publicitaria tratan de hacer operativa la creatividad publicitaria definiendo estrategias para ganar premios a la creatividad (Csikszentmihalyi, 1997; El-Murad y West, 2003; Kover, Goldberg y James, 1995).

Según estos pensamientos, la creatividad consiste en tres elementos fundamentales (Im y Workman, 2004): novedad (Amabile 1983; Amabile 1988; Amabile, 1993), significatividad (Goldenberg, Mazursky y Solomon 1999) y originalidad (Sternberg y Lubart, 1999).

En términos de publicidad, se puede definir novedad como una ruptura relevante de los procesos o costumbres existentes (Ang, Lee y Leong 2007; Ang y Low, 2000). Esto significa 
que la idea general, el mensaje y|la información sobre el producto anunciado debe contener un mensaje singular que sea relevante para el público objetivo (Ang, Lee y Leong 2007; Baack, Wilson y Till 2008; Smith, MacKenzie, Yang, Buchholz y Darley, 2007).

La significatividad debería centrar su atención en la conexión entre el receptor del mensaje del anuncio y el producto anunciado (Sasser y Koslow, 2008).

Kilgour, Sasser y Koslow (2013) reflejaron en su estudio que ganar premios a la creatividad es, principalmente, una función de originalidad. Sin embargo, la originalidad, pese a ser un elemento esencial de la creatividad, no es el único elemento para propiciar respuestas por parte de los consumidores.

Otros numerosos estudios centraron su atención en la influencia de otros factores en la creatividad, como lo son la atmósfera, la motivación y el estilo de liderazgo (Amabile, 1998; Amabile, Conti, Coon, Lazenby y Herron 1996; Amabile, Tighe, Hill y Hennessey, 1994; Blasko y Mokwa 1986; El-Murad y West 2004; Koslow, Sasser y Riordan, 2003; Kover, James y Sonner 1997; Ruscio, Whitney y Amabile, 1997).

La creatividad toma tanta importancia que, incluso, la creatividad es la característica más admirada (Helgesen, 1994; Montano, 2004) en los festivales de publicidad.

Los festivales publicitarios generan prestigio y estatus para las agencias de publicidad (Helgesen, 1994; Schweitzer y Hester, 1992), e indirectamente, para la marca que representa el anuncio, rentabilidad (Tippins y Kunkel, 2006) debida a una excelente creatividad publicitaria.

La cobertura de los festivales de publicidad depende de tres aspectos fundamentales: la cobertura regional, la estructura de sus premios y el objeto de valoración de sus anuncios (Repiso, Berlanga y Lloves, 2015).

Las agencias que quieren participar en sistemas de evaluación acuden a los festivales de publicidad donde la creatividad es especialmente valorada con el objeto de ocupar buenas posiciones en las listas de clasificación (Repiso, Llorente-Barroso y García-García, 2013). 
Los rankings de publicidad tienen una alta repercusión en los medios, en la notoriedad de marca y prestigio de las agencias, ya que sus resultados tienen gran capacidad para contener y simplificar las características de un sistema complejo (Núñez y González, 2015).

Con esta finalidad evaluadora de la creatividad se ha realizado el Ranking Iberoamericano de Publicidad, que evalúa la competitividad de las agencias a través de los resultados creativos de sus anuncios, considerando los premios que reciben de forma ponderada.

Antes de terminar este apartado se hace conveniente mencionar el Código Colombiano de Autorregulación Publicitaria (Comisión Nacional de Autorregulación PublicitariaConarp, 2013). Para las agencias publicitarias colombianas, el interés de este texto radica en que a partir del año 2013 existe un código que indica que los mensajes publicitarios se deben regularizar bajo los principios éticos de responsabilidad social, veracidad, decencia, honestidad, buena fe y transparencia.

Si se hace una lectura tranquila del texto citado en el párrafo anterior, se puede detectar que no figura el concepto de creatividad. Esto mismo sucede en los ordenamientos jurídicos que se están generando actualmente en otras partes del mundo. La mayoría de ellos tienen como objetivo principal, por un lado, proteger legalmente la creatividad publicitaria. Bajo nuestro punto de vista, esta preocupación normativa surge a finales del año 2001 con la aparición de la Declaración Universal de la UNESCO sobre la Diversidad Cultural. $\mathrm{Su}$ artículo 8 establece que:

Ante los cambios económicos y tecnológicos actuales, que abren vastas perspectivas para la creación y la innovación, se debe prestar particular atención a la diversidad de la oferta creativa, al justo reconocimiento de los derechos de los autores y de los artistas, así como al carácter específico de los bienes y servicios culturales que, por ser portadores de identidad, de valores y sentido, no deben ser considerados mercancías o bienes de consumo como los demás. 
Con esta normativa, la UNESCO pretende que los países establezcan a nivel internacional un marco ético y reglamentario destinado a la protección jurídica de la creatividad publicitaria en los medios de comunicación, sobre todo, en las redes sociales.

Y por otro lado, tienen como objetivo minorar la osadía creativa delictiva, engañosa y denigratoria de algunas agencias publicitarias, no solo en la transmisión de sus mensajes (Chalá, 2011) sino también en el desarrollo de sus actividades económicas cuando son contrarias a la dignidad de las personas, la libertad de expresión y a las normas de corrección y buenos usos mercantiles. Se hace conveniente recordar que todos los hechos citados más arriba perjudican de forma muy negativa la imagen corporativa de las empresas. Sin más dilación, se pasa a explicar el proceso para la exploración estadística del lugar que ocupan las agencias de publicidad colombianas dentro del Ranking Iberoamericano de Publicidad durante el periodo comprendido entre los años 2014 y 2016.

\section{METODOLOGÍA}

Como se ha indicado a lo largo de este artículo, el objetivo principal de este trabajo es realizar un análisis de la posición actual de las agencias de publicidad colombianas en el Ranking Iberoamericano de Publicidad, el cual evalúa la calidad de las agencias a través del resultado de su trabajo publicitario y según una ponderación rigurosa de los premios que obtienen y los festivales en las que son galardonadas. Asimismo, el presente trabajo estudia la tendencia según su posición y puntuación en el ranquin desde el año 2014 al 2016. El presente trabajo centra su atención en las primeras 50 del ranquin.

Previamente, se han clasificado los festivales en cuatro categorías que se subdividen en dos categorías: nacional e internacional. En el caso de los festivales nacionales, se han diferenciado los festivales mejor valorados que han sido llamamos "nacional" y "nacional +". Mientras que en el caso de 
los internacionales se han clasificado en dos grupos que han sido llamados "internacional", que agrupa a los festivales europeos y latinoamericanos, e "internacional +", que recopila a los festivales de publicidad mundiales.

La ponderación de los premios se ha realizado utilizando valores similares a los de los festivales. Al premio de mayor categoría (Oro, Gran Premio, etc.) se le ha dado valor 1, y a medida que baja la categoría de los premios se les ha ponderado con valores $2,3,4$ y 5 . Existen festivales que premian de manera extraordinaria uno o dos anuncios con reconocimientos que reflejan el mejor anuncio del año de todas las categorías, como es el caso del premio de Platino del Festival del Sol. A este tipo de premios se le ha dado el valor de 0,5.

El cálculo del valor de las agencias es normalizado (ver fórmula de la figura 1) teniendo en cuenta que para una edición se calcula sobre los premios recibidos en los dos años anteriores, lo que indica que es un ranquin actual.

$$
\text { Agencia }_{z}=\frac{\sum_{i=1}^{m} \sum_{j=1}^{n} \frac{F_{i}}{A n_{j}}+\sum_{x=1}^{p} \sum_{y=1}^{q} \frac{F_{x}}{A n_{y}}{ }^{1 / 2}}{\left(\text { Agencia }_{\alpha}\right)^{1 / 2}}
$$

Fig. 1. Fórmula del ranking de agencias

Fuente: Repiso, Llorente-Barroso \& García-García, 2013

Valor normalizado de la agencia " $z$ " para el periodo 2015 :

$z$ : agencia evaluada

$m$ : festival

$n$ : posición del anuncio dentro del festival

$F_{i}$ : valor del festival en 2013

$A n_{j}$ : valoración del premio recibido en el festival en 2013

$p$ : festival

$q$ : posición del anuncio dentro del festival

$F_{x}$ : valor del festival en 2014

$A n_{y}$ : valoración del premio recibido en el festival en 2014

Se analiza el periodo 2014-2016 para una muestra total de 69, 98 y 73 agencias, respectivamente, en Colombia. 


\section{RESULTADOS}

En este epígrafe se analizará la posición actual y tendencia del periodo 2014 al 2016 de las agencias de publicidad de Colombia en el Ranking Iberoamericano de Publicidad.

El ranquin de agencias para el periodo 2014-2016 muestra las mejores 50 agencias de publicidad en términos de creatividad que participan en el ranquin de Iberoamérica una vez normalizados y ponderados los datos. Como se puede observar en la tabla 1, la agencia Ogilvy \& Mather de Colombia lidera la clasificación tanto en 2014 como en 2015, aunque, en 2016, Sancho $B B D O$ lidera el listado seguida de la agencia Lowe SSP3, que también mantiene su segundo puesto en ambos periodos.

Concretamente, en 2014, Ogilvy \& Mather Brasil, con la primera posición, se mantiene en 2015 , aunque desciende a la tercera posición en 2016 .

Entre las agencias que mantienen su posicionamiento encontramos que Lowe SSP3, Leo Burnett Colombia, Young and Rubicam Colombia y DDB Colombia se encuentran en segunda, cuarta, sexta y octava posición en 2014 y se mantiene en 2016. Asimismo, la agencia Jairo Enrique Bonilla Mayorga mantiene su puesto 45 tanto en 2014 como en 2016.

Las agencias que han ganado posicionamiento en el ranquin han sido Sancho BBDO en tercera posición; McCann Erickson Colombia en séptima; Glue en décima posición; Momentum Colombia en el puesto 17; Treetop Rocket en el 18; TbwaColombia en el 26; Do! Advertainment en el 31; Rep/Grey en el 33; Txt Agencia de Marca en el 34; Brm en la posición 36 y Jwt Colombia en el puesto 38 mejoran su posicionamiento para 2016, alcanzando el puesto 1, 5, 7, 11, 9, 18, 19, 30, 24, 25 y 28, respectivamente.

Por su parte, entre las agencias que pierden posición en el ranquin de 2014 a 2016 pero se mantienen dentro de las primeras 50, se encuentra BTL (de la posición 5 a la 30), Proximity Colombia (de la 9 a la 12), On Brand Experience (de la 11 a la 14), Sistole SA (de la 12 a la 49), Publicis Colombia (de la 13 a la 17), Sphera Impacta (de la 21 a la 51), Netbangers (de la 24 a la 51), Aguayo Publicidad (de la 25 a la 27), PHD Colombia (de la 30 a la 38), ID Interactive (de la 37 a la 45), Coctelbrand (de la 43 a la 58) y Planeta (de la 43 a la 45). 


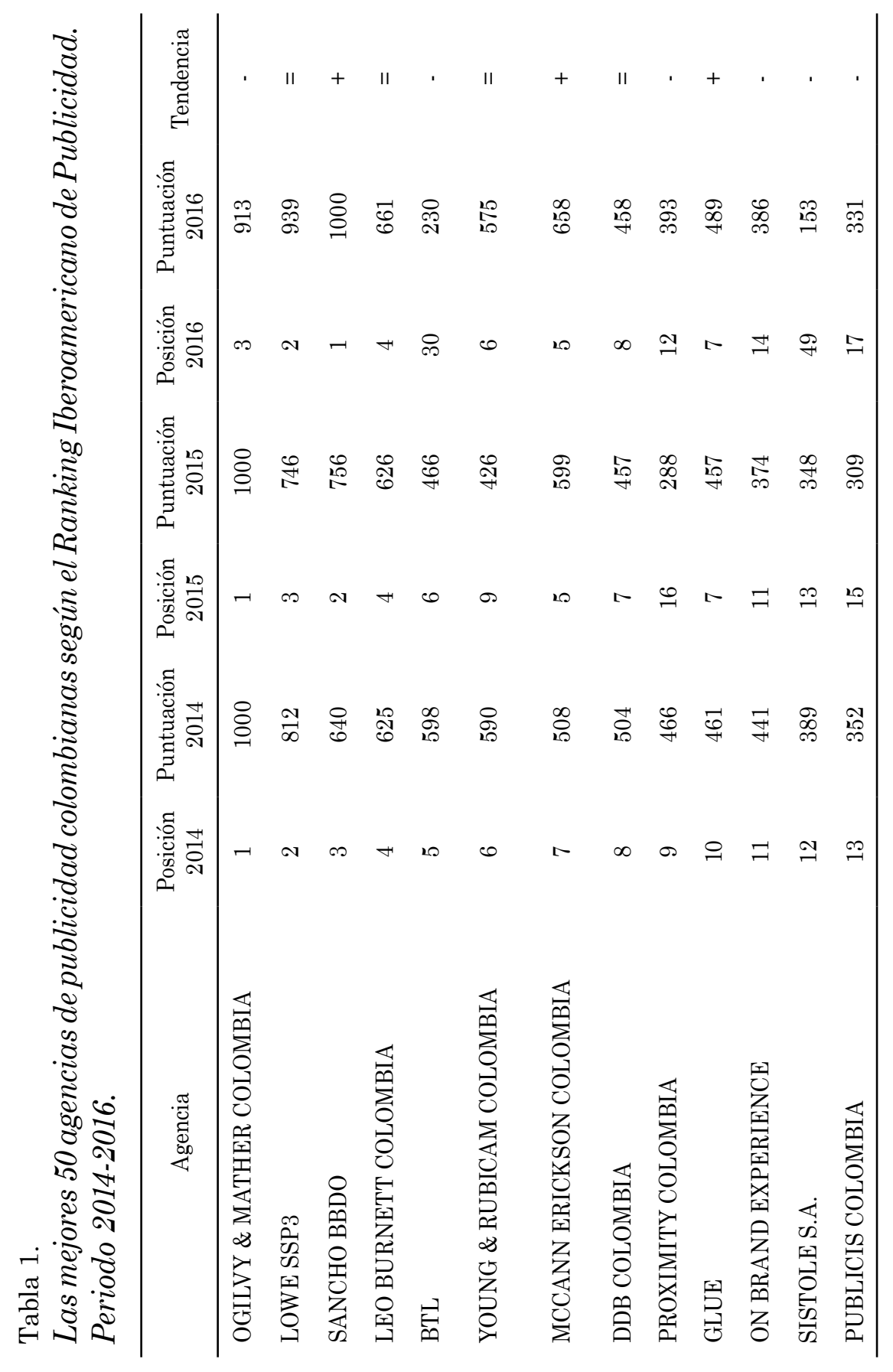




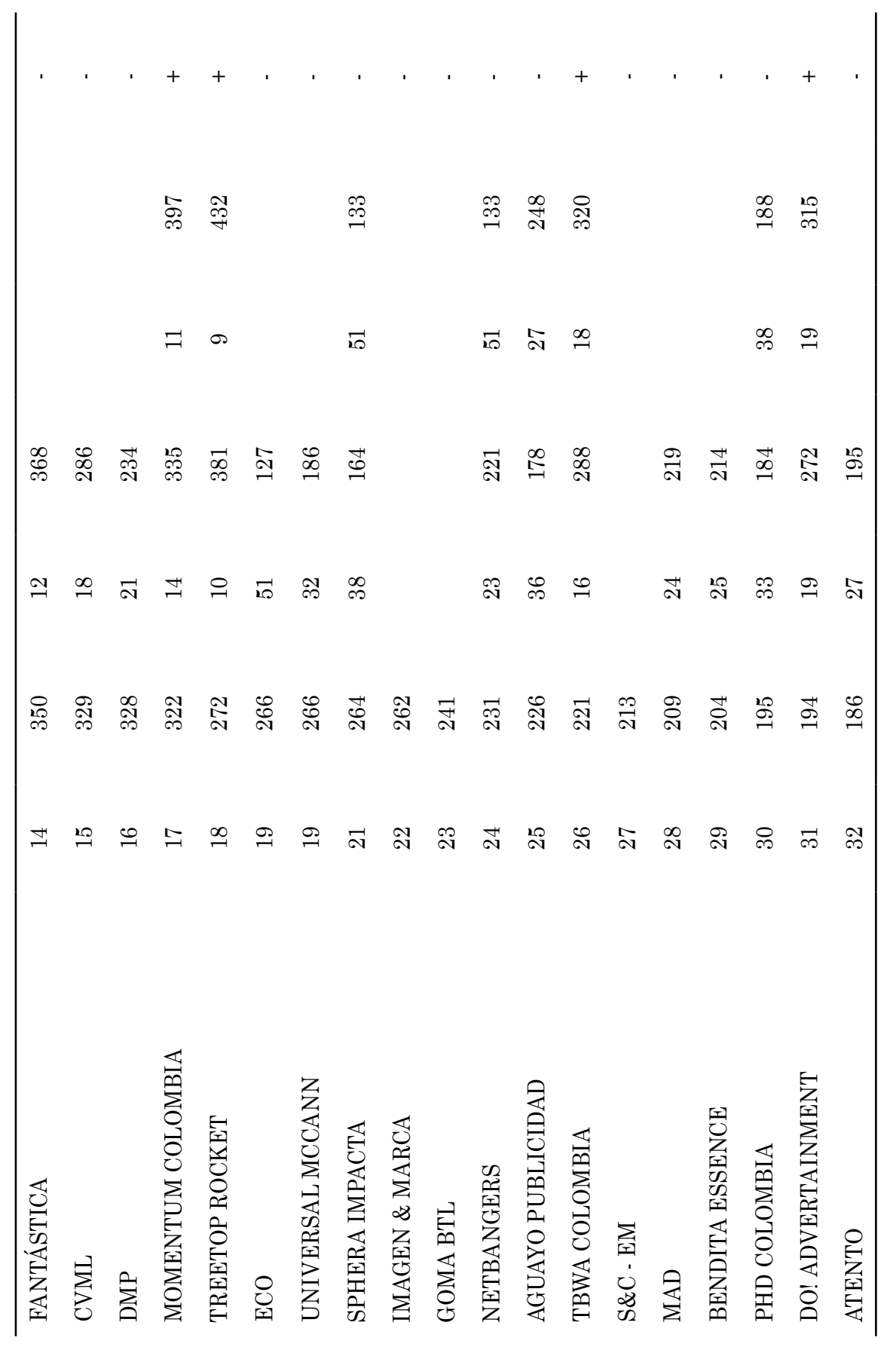




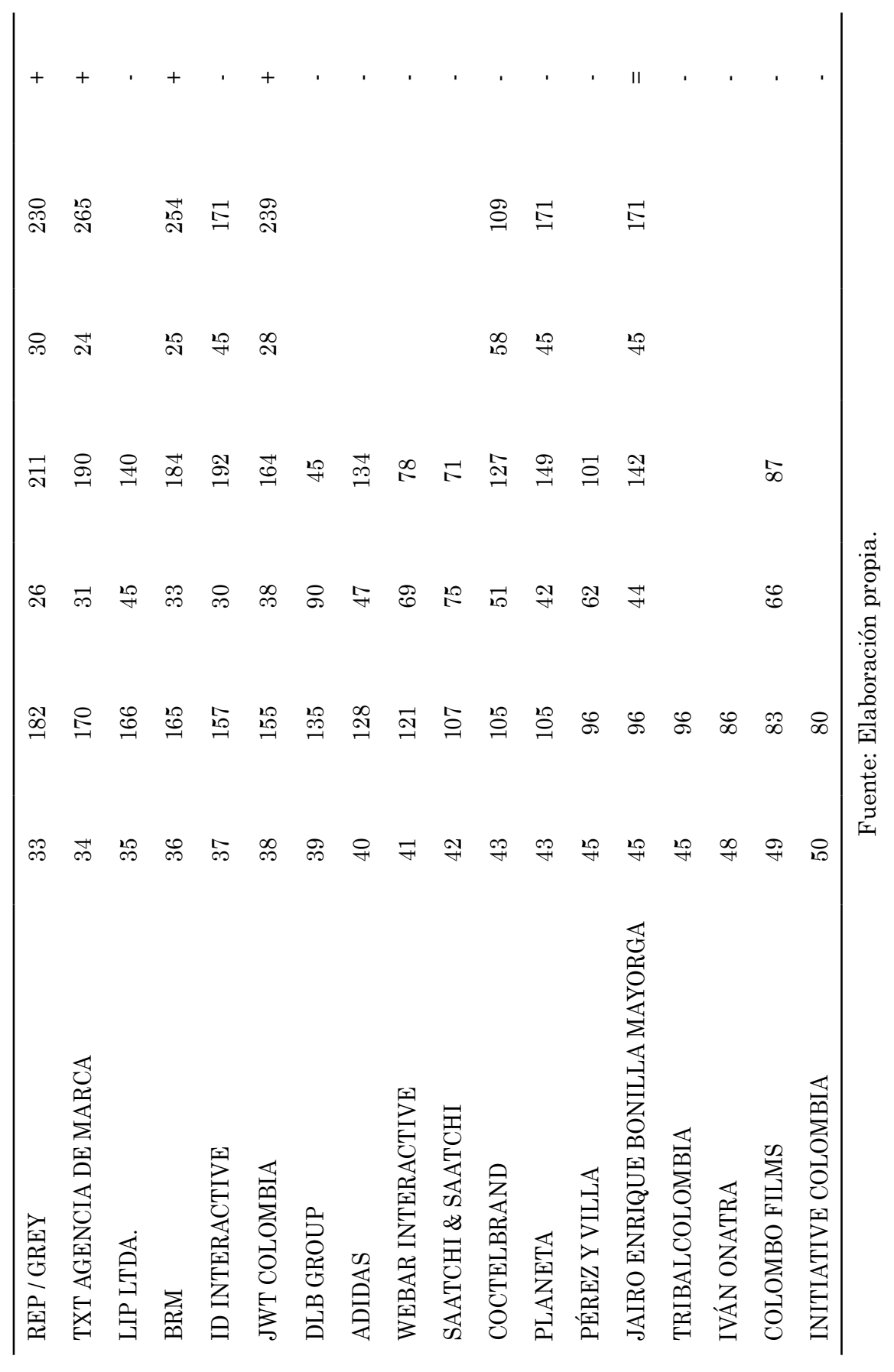


Finalmente, las agencias con tendencia negativa (ya que han perdido puestos en el ranquin saliendo del posicionamiento de las mejores 50) han sido Fantástica, Cuml, Dmp, Eco, Universal McCann, Imagen \& Marca, Goma BTL, S\&CEM, MAD, Bendita Essence, Atento, Lip Ltda., Dlb group, Adidas, Webar Interactive, Saatchi \& Saarchi, Pérez y Villa, Tribalcolombia, Iván Onatra, Colombo Films, e Interactive Colombia.

\section{CONCLUSIONES}

El presente trabajo ha intentado hacer una primera aproximación al sector de la publicidad a través de la descripción de las posiciones obtenidas por las agencias en el Ranking Iberoamericano de la Publicidad. Se han estudiado en concreto las 50 agencias más creativas de Iberoamérica. Si se toman en cuenta los resultados mostrados en la tabla 1, se puede afirmar que Ogilvy \& Mather, Lowe SSP3 y Sancho $B B D O$ lideran el ranquin con su posicionamiento en las tres primeras posiciones en 2014, 2015 y 2016.

Por su parte, de la edición 2014 a 2016, las 10 mejores agencias son altamente creativas en los tres años analizados, ya que mantienen su posicionamiento, o incluso, lo mejoran, y aunque alguna pierda puestos, se sigue manteniendo dentro de las 10 agencias más creativas. Estas agencias son: Ogilvy \& Mather Colombia, Lowe SSP3, Sancho BBDO, Leo Burnett Colombia, BTL, Young \& Rubicam Colombia, Mccann Erickson Colombia, DDB Colombia, Proximity Colombia y Glue.

El ranquin analizado, además de ordenar y estudiar la tendencia de las agencias por su calidad creativa, es un buen referente para estudiar la publicidad en Colombia y servir como impulsor de la competitividad.

Asimismo, se observa que las agencias mejor situadas son las internacionales. Desde la perspectiva jurídica, este artículo ha detectado que una de las razones de este hecho se encuentra en que las grandes agencias que rigen la publici- 
dad en Iberoamérica, Europa y Estados Unidos (donde están insertas las más creativas), se caracterizan porque tienen un modelo de gobernanza que garantiza, por un lado, el cumplimiento legal de los valores éticos que establecen los diferentes códigos de autorregulación publicitaria donde desarrolla su actividad económica, y por otro, a que la difusión de sus mensajes publicitarios se efectúa conforme a conductas socialmente responsables. Este estudio pone de manifiesto que esa práctica gerencial, cimentada en la aplicación positiva de los ordenamientos publicitarios, contribuye significativamente en la competitividad empresarial de las agencias publicitarias colombianas durante el marco temporal objeto de nuestra investigación.

Como limitación a este estudio, se debe señalar que solo son analizadas aquellas agencias que participan en los festivales señalados. Por tanto, las agencias que no participan en dichos festivales se quedan fuera. Autores como Polonsky y Waller (1995) indican que la razón para que no participen es porque la creatividad de sus campañas publicitarias es pobre en relación con las de sus competidores.

También se debe señalar que la mayoría de los clientes en la actualidad requieren de sus agencias la creación de ideas creativas que no solo apunten a un canal de comunicación sino a todos los posibles canales (Kotler y Keller, 2006; Naik y Raman, 2003).

En definitiva, el presente trabajo contribuye a la mejora constante de la formación profesional del comunicador colombiano, dando una visión del mercado de las agencias publicitarias más creativas que ofrecen posibilidades de exposición a mercados internacionales.

Para las agencias de publicidad, la mejor herramienta de promoción en la industria publicitaria actual es obtener premios a la creatividad y conseguir posicionarse entre las primeras agencias del Ranking de Publicidad de Iberoamérica. Este hecho permite incrementar la fuente de ingresos y la competitividad (Wentz, 2005). 


\section{REFERENCIAS}

Amabile, T. M. (1988). A model of creativity and innovation in organitations, Research in organitation behavior, 10, 123167.

Amabile, T. M. (1998). How to Kill Creativity. Harvard Business Review, 76(5), 76-86.

Amabile, T. M., Conti R., Coon, H., Lazenby, J. y Herron, M. (1996). Assessing the Work Environment for Creativity. Academy of Management Journal, 39(5), 1154-1184.

Andrews, J. C. y Smith, D. C. (1996). In Search of the Marketing Imagination: Factors Affecting the Creativity of Marketing Programs for Mature Products. Journal of Marketing Research, 33(2), 174-187.

Ang, S. H., Lee, Y. H. y Leong, S. M. (2007). The ad creativity cube: Conceptualization and initial validation. Journal of the Academy of Marketing Science, 35(2), 220-232.

Ang, S. H. y Low, S. Y. (2000). Exploring the dimensions of ad creativity. Psychology \& Marketing, 17(10), 835-854.

Baack, D. W., Wilson, R. T. y Till, B. D. (2008). Creativity and memory effects: Recall, recognition, and an exploration of nontraditional media. Journal of Advertising, 37(4), 85-94.

Barron, F. (1955). The Disposition toward Originality. Journal of Abnormal Psychology, 51(3), 478-485.

Barron, F. y Harrington, D. M. (1981). Creativity, intelligence, and personality. Annual review of psychology, 32(1), 439476.

Bell, J. A. (1992). Creativity, TV Commercial Popularity, and Advertising Expenditures. International Journal of Advertising, 11(2), 165-183.

Bergkvist, L. y Rossiter, J. R. (2008). The role of ad likability in predicting an ad's campaign performance. Journal of advertising, 37(2), 85-98.

Besemer, S. y O'Quin, K. (1986). Analyzing creative products: Refinement and test of a judging instrument. The Journal of Creative Behavior, 20(2), 115-126.

Besemer, S. P. y Treffinger, D. J. (1981). Analysis of Creative Products: Review and Synthesis. The Journal of Creative Behavior, 15(3), 158-178. 
Buzzell, R. D. (1964). Predicting Short-Term Changes in Market Share as a Function of Advertising Strategy. Journal of Marketing Research, 1(3), 27-31.

Bogart, L., Tolley, B. S. y Orenstein, F. (1970). What one little ad can do. Journal of Advertising research, 10(4), 3-13.

Bruner, J. (1962). "The Conditions of Creativity". En Gruber, H.E., Terrell G. E. y Wertheimer, M. (eds.), Contemporary Approaches to Creative Thinking. Nueva York: Atherton Press.

Butkys, A. S. y Herpel, G. (1992). How advertising agencies handle their own advertising strategy: an industry-wide overview of its self-promotion efforts. Journal of Advertising Research, 32, 18-22.

Chalá, P. (2011). La legislación como condicionante en la creatividad. Barcelona: Universidad Autónoma de Barcelona.

Comisión Nacional de Autorregulación Publicitaria - Conarp (2013). Código Colombiano de Autorregulación Publicitaria. Bogotá: Comisión Nacional de Autorregulación Publicitaria.

Dahlen, M., Rosengren, S. y Törn, F. (2008). Advertising creativity matters. Journal of Advertising Research, 48(3), 392-403.

Deshpande, R., Farley, J. U. y Webster Jr, F. E. (1993). Corporate culture, customer orientation, and innovativeness in Japanese firms: a quadrat analysis. The journal of Marketing, 57(1), 23-37.

El-Murad, J. y West, D. C. (2003). Risk and creativity in advertising. Journal of Marketing Management, 19(5-6), 657-673.

Haberland, G. S. y Dacin, P. A. (1992). The Development of a Measure to Assess Viewers' Judgments of the Creativity of an Advertisement. Advances in Consumer Research, 19(1), 817-825.

Helgesen, T. (1994). Advertising awards and advertising agency performance criteria. Journal of Advertising Research, (34), 43-43.

Im, S. y Workman Jr, J. P. (2004). Market orientation, creativity, and new product performance in high-technology firms. Journal of marketing, 68(2), 114-132.

Jackson, P. W. y Messick, S. (1965). The person, the product, and the response: conceptual problems in the assessment of creativity. Journal of personality, 33(3), 309-329. 
Jones, J. P. (1995). Single-Source Research Begins to Fulfill its Promise. Journal of Advertising Research, 35(3), 9-16.

Gibson, L. D. (1996). What Can One TV Exposure Do? Journal of Advertising Research, 36(2), 9-18.

Goldenberg, J., Mazursky, D. y Solomon, S. (1999). Creative sparks. Science, 285(5433), 1495-1496.

Kilgour, A. M., Sasser, S. y Koslow, S. (2013). Creativity awards: Great expectations? Creativity Research Journal, 25(2), 163-171.

Kübler, R.V. y Proppe, D. (2012). Faking or Convincing: Why Do Some Advertising Campaigns Win Creativity Awards? BuR - Business Research, 5(1) 60-81.

Li, H., Dou, W., Wang, G. y Zhou, N. (2008). The effect of agency creativity on campaign outcomes: The moderating role of market conditions. Journal of Advertising, 37(4), 109-120.

MacKinnon, D. W. (1987). "Some Critical Issues for Future Research". En Isaksen, S.G., (ed.), Creativity: Frontiers of Creativity Research: Beyond the Basics. Nueva York: Bearly Ltd.

Michell, P. C. (1984). Accord and Discord in Agency-Client Perceptions of Creativity. Journal of Advertising Research, 24(5), 9-24.

Montano, J. R. (2004). Characteristics of U.S. Hispanic Advertising. A comparison of awards winning and Non-awardwinning Commercial. [Tesis doctoral inedita]. Universidad de Florida: Gainesville.

Moorman, C. y Miner, A. S. (1997). The impact of organizational memory on new product performance and creativity. Journal of marketing research, 91-106.

Mumford, M. D. y Simonton, D. K. (1997). Creativity in the workplace: People, problems, and structures. The journal of creative behavior, 31(1), 1-6.

Myers, J. (2004). Advertising Accountability, R-o-I \& SarbanesOxley Concerns Spawn Audit Industry. Recuperado el 14 de enero de 2008 de: https://www.mediavillage.com/author/ jack-myers/\#page $=168 \&$ tab=latest

Naik, P. A. y Raman, K. (2003). Understanding the impact of synergy in multimedia communications. Journal of Marketing Research, 40(4), 375-388. 
Newell, A., Shaw, J. C. y Simon, H. A. (1959). The processes of creative thinking. Santa Monica, CA: Rand Corporation.

Núñez, E. y González, A. (2015). Ranking de anuncios y agencias de publicidad en Venezuela. Opción, 31(Especial 3), 907-921.

Ogilvy, D. (1963). Confessions of an Advertising Man. Nueva York: Atheneum.

Pieters, R., Warlop, L. y Wedel, M. (2002). Breaking through the clutter: Benefits of advertisement originality and familiarity for brand attention and memory. Management Science, 48(6), 765-781.

Polonsky, M. J. y Waller, D. S. (1995). Does winning advertising awards pay?: The Australian experience. Journal of Advertising Research, 35(1), 25-25.

Repiso, R., Berlanga, I. y Lloves, B. (2015). Ranking de anuncios y agencias de publicidad en Chile. Revista F@ ro: revista teórica del Departamento de Ciencias de la Comunicación, 1(21), 36-52.

Repiso, R., Llorente-Barroso, C. y García-García, F. (2013). Ranking ESCO de agencias de publicidad: Descripción y resultados. El profesional de la información, 22(2), 181-187.

Rossiter, J. R. (2008). Defining the Necessary Components of Creative, Effective Ads. Journal of Advertising, 37 (4), 139144.

Sasser, S. L. y Koslow, S. (2008). Desperately seeking advertising creativity: Engaging an imaginative "3Ps" research agenda. Journal of Advertising, 37(4), 5-20.

Schweitzer, J. C. y Hester, J. B. (1992). The importance of winning advertising award shows. Southwestern Mass Communication, 7(1), 55-66.

Sethi, R., Smith, D. C. y Park, C. W. (2001). Cross-functional product development teams, creativity, and the innovativeness of new consumer products. Journal of Marketing Research, 38(1), 73-85.

Smith, R. E., MacKenzie, S. B., Yang, X., Buchholz, L. M. y Darley, W. K. (2007). Modeling the determinants and effects of creativity in advertising. Marketing science, 26(6), 819-833.

Sternberg, R. J. y Lubart, T. (1999). The Concept of Creativity: Prospects and Paradigms. En Sternberg, R. J. (ed.), Handbook of Creativity. Cambridge: Cambridge University Press. 
Stone, G., Besser, D. y Lewis, L. E. (2000). Recall, liking, and creativity in TV commercials: A new approach. Journal of Advertising Research, 40(3), 7-18.

Till, B. D. y Baack, D. W. (2005). Recall and Persuasion: does creative advertising matter? Journal of Advertising, 34(3), 47-57.

Tippins, M. J. y Kunkel, R. A. (2006). Winning a Clio advertising award and its relationship to firm profitability. Journal of Marketing Communications, 12(1), 1-14.

Wentz, L. (2005). At Cannes, the Lions Say "Grrr". Advertising Age, 76 (26), 1-2. 